\title{
Novel biomarkers of acute kidney injury: time for implementation?
}

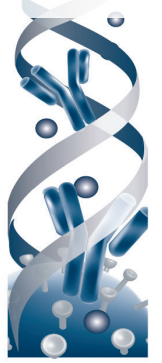

Keywords: AKI biomarker discovery $\bullet$ AKI biomarkers in clinical trials $\bullet$ AKI diagnostic matrix - biomarker cutoffs $\bullet$ triaging with biomarkers of AKI

Acute kidney injury (AKI) is associated with significant early and late [1] mortality, even after 10 years and regardless of the degree of functional recovery [2]. After AKI, 7.8 subjects per 100 patient years develop chronic kidney disease and 4.9 per 100 patient years proceed to end-stage renal disease [3]. Since our demonstration in 2003 that urinary biomarkers collected on admission to an intensive care unit could predict development of AKI [4], an ever increasing number of novel biomarkers of kidney damage have emerged. Most of these novel biomarkers and the older cluster of tubular enzymes are markers of structural injury, in other words, 'renal damage biomarkers'. They have been used for AKI risk assessment, early diagnosis, and prognosis, and are potentially useful for differential diagnosis, and for monitoring the course of injury and response to therapy. This has been recognized and incorporation into definitions of AKI has been recommended by both the Acute Dialysis Quality Initiative group [5] and by regional practice guidelines in Australia and New Zealand [6].

Incorporation of damage biomarkers into clinical practice awaits agreement regarding the appropriate thresholds (cut-offs) for diagnosis or categories of severity. As noted [7], such thresholds are contextual and can vary with cause and context of AKI. As measured by the area under the receiver operator curve (AUC), biomarker performance varies considerably, particularly in heterogeneous populations [8]. However, as highlighted by Kellum and Devarajan in this issue [9], even a modestly performing biomarker with an
AUC less than 0.70 can assist in clinical decision making, simply by selecting an appropriate cutoff. Given the poor utility of serum creatinine in AKI, they argue further that it is already imperative that these biomarkers are used in clinical trials to enrich the target population for interventions, with the obvious critically needed benefit of reducing the number needing to be enrolled. Clearly, the same considerations apply whether triaging with biomarkers is used to identify AKI or recovery from AKI [10].

Biomarker threshold selection requires assay standardization and identification of normal reference intervals. Commercialization helps drive assay standardization and assays for the best performed novel biomarkers, neutrophil gelatinase-associated lipocalin (NGAL) and kidney injury molecule-1 (KIM-1) are widely available. Only one published study has examined the effects of different storage conditions on urinary biomarker concentrations [11]. Reference intervals for novel biomarkers are not widely available and current trials have used different strategies for defining these after the fact. For instance, we demonstrated that subjects with so-called 'pre-renal' AKI had mildly elevated urinary KIM-1, IL-18 and cystatin C compared with subjects who did not develop an increase in serum creatinine at any time [12]. These observations were confirmed by Doi et al. [13] who observed increases in urinary L-type fatty acid binding protein (L-FABP), NGAL, IL-18, $N$-acetyl$\beta$-D-glucosaminidase (NAG) and albumin in similarly defined subjects in an intensive

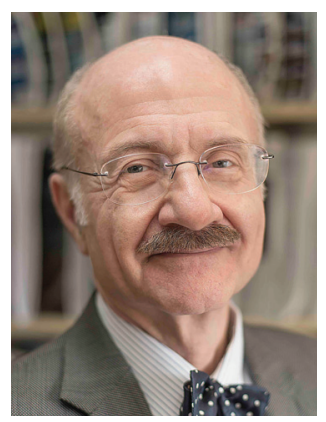

Zoltan H Endre

Department of Nephrology, Prince of Wales Hospital, High St, Randwick, Sydney, NSW, Australia and

Prince of Wales Clinical School, University of New South Wales, Sydney, NSW, Australia

zoltan.endre@unsw.edu.au 
care unit. These observations confirm that transient increases in serum creatinine in critically ill subjects are associated with increases in damage biomarkers and highlight that prerenal AKI is merely the mild end of a continuous spectrum of severity of AKI.

Up to now, biomarker cutoffs have been validated against serum creatinine, since absolute or relative increases in creatinine are the primary basis for the consensus definitions of AKI, although the changes actually are surrogates for change in GFR [14]. While a biomarker increase can predict an increase in creatinine, only the eventual change in creatinine can diagnose AKI. This circular argument ignores the wellknown deficiencies of creatinine and suggests further that only changes in function are important. Hence an alternative GFR surrogate to serum creatinine, such as serum cystatin $\mathrm{C}$ might be substituted and damage biomarker prediction assessed against cystatin $\mathrm{C}$ based diagnosis of AKI. This too is illogical.

The beauty of the matrix of damage and function markers recommended for diagnosis by the ADQI group [5] is that either damage or function marker change or both can be used for diagnosis. However for this to be useful, the cutoffs for functional and damage markers in the ADQI AKI matrix need to be derived against an independent gold standard. Since dialysis requirement or death are significant clinical outcomes of AKI, we proposed a method for establishing biomarker cutoffs by selecting values that had the same sensitivity for these outcomes as serum creatinine [15]. This method was examined in a total of 507 subjects and generated values for absolute urinary biomarker concentrations that are similar to those revealed for NGAL by a metanalysis of observational studies in cardiorenal AKI [16] and for both NGAL and KIM-1 in a large cohort screened in the emergency department [17]. While the values require validation in large cohorts, the method is robust and will allow classification, staging and differential diagnosis of AKI using the damage function matrix recommended by ADQI. This or similar approaches that define cut-offs independent of serum creatinine are needed for the prospective validation of the biomarker-positive, creatinine-negative category of AKI and to allow evolution to injury biomarker-based triaging of patients outside the domain of clinical research.

One study has highlighted the GFR dependence of plasma NGAL levels [18]. In this issue, McWilliam et al. [19] report reference intervals for urinary NGAL and KIM-1 in healthy children in both United States and United Kingdom. They identified that NGAL was consistently higher in females than males and ethnic differences and diurnal variation in urinary KIM-1 concentrations. Studies also reported in this issue by
Mohammadjafari [20] demonstrate that in neonates with an antenatal diagnosis of hydronephrosis, urinary KIM-1 and epidermal growth factor levels identify those with severe obstruction. A study in Poland [21] has demonstrated that male, obese, hypertensive adolescents with hyperuricemia have higher urine NGAL and KIM-1 levels relative to normouricemia adolescents. Taken together, these studies highlight the urgent need to define urinary and plasma damage biomarker reference intervals in diverse healthy populations.

Variation in biomarker performance nevertheless allows room for even newer biomarkers that might perform better in both selective and heterogeneous populations. Biomarkers that are highly selective for target populations are needed to facilitate individualization of patient care: so-called 'theragnostics' discussed briefly by Kellum and Devarajan in this issue [9]. On the other hand, identification of discriminating biomarkers from heterogeneous (instead of homogenous) populations leads to novel biomarkers that can be used more widely in less well-defined subjects. For example, the excellent performance of new urinary biomarkers, the cell cycle inhibitors, namely, tissue inhibitor of matrix metalloproteinase, TIMP-2, and insulin-like growth factor-binding protein, IGF7BP7, has been validated in the heterogeneous setting of critically ill patients [22]. These more recently identified biomarkers of damage may be better performed because they are more sensitive and less selective and thus respond to a wider variety of renal insults than earlier novel biomarkers selected on the presumed basis of a single mechanism of renal injury.

New novel biomarkers, new uses for existing markers and ways of identifying further biomarkers are discussed in several papers in this issue. Haase et al. recently used an antibody-free proteomic approach to identify three novel biomarkers of kidney function, fibrosis and ischemic tissue damage, namely, LG3 (a C-terminal fragment of perlecan), latent transforming growth factor binding protein-2, LTBP-2 (an extracellular matrix protein) and cathepsin $\mathrm{L}$, a ubiquitously expressed cysteine endopeptidase. In the present study, Haase et al. [23] use MS analysis to demonstrate in patients undergoing open heart surgery, that at differing times, LG3, LTPBP2, cathepsin L as well as NGAL and cystatin $\mathrm{C}$ had greater predictive value for AKI and major adverse kidney events than creatinine, urea and urine output. Fernando et al. [24] review calcineurin inhibitor (CNI) metabolism and discuss how various methods, particularly Omics strategies, have revealed potential biomarkers that might distinguish CNI nephrotoxicity from rejection or ischemic injury in renal transplant recipients. Che et al. [25] demonstrate that mildly reduced serum bicarbonate, both 
alone or in combination with serum creatinine predict poorer long-term renal function after an episode of AKI. Finally, because of the importance of catecholamine metabolism in the setting of cardiac surgery, Albert et al. [26] examined the association between genotypes of catecholamine-O-methyltransferase and AKI in 200 adult patients undergoing cardiopulmonary bypass; importantly the authors utilized both serum creatinine and the urinary damage biomarkers NGAL and midkine to test these associations.

We are fortunate to be in the biomarker era. After decades of delay and uncertainty in diagnosis of AKI, there now exist methods for early detection, differential diagnosis and for monitoring renal status that are independent of serum creatinine. This collection of papers addresses critical issues in discovery and implementation of renal biomarkers in clinical practice and research. While optimization and cost remain ongoing concerns, the most salient conclusion from these

\section{References}

1 Chawla LS, Amdur RL, Shaw AD, Faselis C, Palant CE,

Kimmel PL. Association between AKI and long-term renal and cardiovascular outcomes in United States veterans. Clin. J. Am. Soc. Nephrol. 9(3), 448-456 (2014).

2 Hobson CE, Yavas S, Segal MS et al. Acute kidney injury is associated with increased long-term mortality after cardiothoracic surgery. Circulation 119(18), 2444-2453 (2009).

3 Coca SG, Yusuf B, Shlipak MG, Garg AX, Parikh CR. Longterm risk of mortality and other adverse outcomes after acute kidney injury: a systematic review and meta-analysis. Am. J. Kidney Dis. 53(6), 961-973 (2009).

4 Westhuyzen J, Endre ZH, Reece G, Reith DM, Saltissi D, Morgan TJ. Measurement of tubular enzymuria facilitates early detection of acute renal impairment in the intensive care unit. Nephrol. Dial. Transplant 18(3), 543-551 (2003).

5 Murray PT, Mehta RL, Shaw A et al. Current use of biomarkers in acute kidney injury: report and summary of recommendations from the 10th Acute Dialysis Quality Initiative consensus conference. Kidney Int. 85(3), 513-521 (2014).

6 Langham RG, Bellomo R, D' Intini V et al. KHA-CARI guideline: KHA-CARI adaptation of the KDIGO Clinical Practice Guideline for Acute Kidney Injury. Nephrology 19(5), 261-265 (2014).

7 Endre ZH, Pickering JW. Biomarkers and creatinine in AKI: the trough of disillusionment or the slope of enlightenment? Kidney Int. 84(4), 644-647 (2013).

8 Endre ZH, Pickering JW, Walker RJ et al. Improved performance of urinary biomarkers of acute kidney injury in the critically ill by stratification for injury duration and baseline renal function. Kidney Int. 79(10), 1119-1130 (2011).

9 Kellum JA, Devarajan P. What can we expect from biomarkers for acute kidney injury? Biomarkers Med. 8(10), 1239-1245 (2014). studies, is that, we can no longer afford not to use biomarkers in risk stratification for clinical trials. Furthermore, we need to take further note of the lessons learned by our colleagues in cardiology, who have focused on 'how' not 'whether' to use damage biomarkers in myocardial ischemic injury. Selected damage biomarkers should be starting to enter routine clinical practice in parallel with functional markers wherever possible.

\section{Financial \& competing interests disclosure}

ZH Endre has received honoraria as a member of the Novartis Australia Medical Advisory Board. The author has no other relevant affiliations or financial involvement with any organization or entity with a financial interest in or financial conflict with the subject matter or materials discussed in the manuscript apart from those disclosed.

No writing assistance was utilized in the production of this manuscript.

10 Endre Z. Recovery from acute kidney injury: the role of biomarkers. Nephron. Clin. Pract.127(1-4) 101-105 (2014).

11 Parikh CR, Butrymowicz I, Yu A et al. Urine stability studies for novel biomarkers of acute kidney injury. Am. J. Kidney Dis. 63(4), 567-572 (2014).

12 Nejat M, Pickering JW, Devarajan P et al. Some biomarkers of acute kidney injury are increased in pre-renal acute injury. Kidney Int. 81(12), 1254-1262 (2012).

13 Doi K, Katagiri D, Negishi K et al. Mild elevation of urinary biomarkers in prerenal acute kidney injury. Kidney Int . 82(10), 1114-1120 (2012).

14 Endre ZH, Pickering JW, Walker RJ. Clearance and beyond: the complementary roles of GFR measurement and injury biomarkers in acute kidney injury (AKI). Am. J. Physiol. Renal Physiol. 301, F697-F707 (2011).

15 Pickering JW, Endre ZH. Linking injury to outcome in acute kidney injury: a matter of sensitivity. PLoS ONE 8(4), e62691 (2013).

16 Haase M, Devarajan P, Haase-Fielitz A et al. The outcome of neutrophil gelatinase-associated lipocalin-positive subclinical acute kidney injury a multicenter pooled analysis of prospective studies. J. Am. Coll. Cardiol. 57(17), 1752-1761 (2011).

17 Nickolas TL, Schmidt-Ott KM, Canetta P et al. Diagnostic and prognostic stratification in the emergency department using urinary biomarkers of nephron damage: a multicenter prospective cohort study. J. Am. Coll. Cardiol. 59(3), 246-255 (2012).

18 Donadio C. Effect of glomerular filtration rate impairment on diagnostic performance of neutrophil gelatinaseassociated lipocalin and B-type natriuretic peptide as markers of acute cardiac and renal failure in chronic kidney disease patients. Crit. Care 18(1), R39 (2014).

19 McWilliam S, Antoine D, Sabbisetti V et al. Reference Intervals for urinary renal injury biomarkers, kidney injury molecule-1 (KIM-1) and neutrophil gelatinase-associated 
lipocalin (NGAL), in healthy children Biomarkers Med. 8(10), 1189-1198 (2014).

20 Mohammadjafari H. Determination of the severity of ureteropelvic junction obstruction using urinary epidermal growth factor and kidney injury molecule 1 levels. Biomarkers Med. 8(10), 1199-1206 (2014).

21 Tomczak J, Wasilewska A, Milewski R. Urine NGAL and KIM-1 in children and adolescents with hyperuricemia. Pediatr. Nephrol. 28(9), 1863-1869 (2013).

22 Kashani K, Al-Khafaji A, Ardiles T et al. Discovery and validation of cell cycle arrest biomarkers in human acute kidney injury. Crit Care. 17(1), R25 (2013).
23 Haase M, Bellomo R, Albert C et al. The identification of three novel biomarkers of major adverse kidney events. Biomarkers Med. 8(10), 1207-1217 (2014).

24 Fernando M, Peake P, Endre Z. Biomarkers of calcineurin inhibitor nephrotoxicity in transplantation. Biomarkers Med. 8(10), 1247-1262 (2014).

25 Che X, Xie Y, Wang C et al. Blood $\mathrm{HCO}_{3}{ }^{-}$concentration predicts the long-term prognosis of acute kidney injury patients. Biomarkers Med. 8(10), 1219-1226 (2014).

26 Albert C, Kube J, Haase-Fielitz A et al. Pilot study of association of catechol-O-methyl transferase rs 4680 genotypes with acute kidney injury and tubular stress after open heart surgery. Biomarkers Med. 8(10), 1227-123 (2014). 